\title{
Topological Insulator in an Atomic Liquid
}

\author{
Gia-Wei Chern ${ }^{1}$ \\ ${ }^{1}$ Department of Physics, University of Virginia, Charlottesville, VA 22904, USA
}

(Dated: September 28, 2018)

\begin{abstract}
We demonstrate theoretically an atomic liquid phase that supports topologically nontrivial electronic structure. A minimum two-orbital model of liquid topological insulator in two dimensions is constructed within the framework of tight-binding molecular dynamics. As temperature approaches zero, our simulations show that the atoms crystallize into a triangular lattice with nontrivial band topology at high densities. Thermal fluctuations at finite temperatures melt the lattice, giving rise to a liquid state which inherits the nontrivial topology from the crystalline phase. The electronic structure of the resultant atomic liquid is characterized by a nonzero Bott index. Our work broadens the notion of topological materials, and points to a new systematic approach for searching topological phases in amorphous and liquid systems.
\end{abstract}

Topological insulators (TI) are a class of free or weaklyinteracting fermionic systems that exhibit topologically nontrivial electron bands 1, 2. One intriguing manifestation of the nontrivial band topology is the appearance of gapless electronic states confined to the sample surface. The study of TI started with the integer quantum Hall (QH) effect, where the Hall conductance is quantized in integer multiples of the universal conductance 3 . It was later shown that this quantization is related to a topological index called the TKNN or Chern number of the occupied bands [4]. Recently, renewed interest in TI was triggered by the discovery of time-reversal invariant systems characterized by a $Z_{2}$ index [7. These include the two-dimensional (2D) quantum spin-Hall insulators $8-11$ and 3D topological insulators 12 15. Conventionally, the definition of topological indexes assumes a well-defined Brillouin zone (BZ) from a perfect lattice. For example, the Chern number of TI can be understood as a generalized winding number characterizing the mapping from the BZ to the Hilbert space of Bloch states $[5$. Similarly, definitions of the $Z_{2}$ invariant mostly rely on the condition that Bloch momentum is a good quantum number [12 14. Such approaches have enabled a complete classification of TI [16-19] based on the ten symmetry classes of Hamiltonians introduced in the seminal work of Altland and Zirnbauer [20].

Robustness against disorder is another defining feature of TI. Effects of disorder on QH insulator as well as TI have been extensively investigated $21-27$. Generally speaking, topological properties, such as quantized conductance and gapless boundary modes, are robust against disorder that preserves certain discrete symmetries of the symmetry class, and importantly, provided that the spectral or mobility gap remains finite. Significant efforts have also been devoted to understand disorder-induced non-Anderson-type transitions 28 34. The nontrivial interplay between disorder and band topology also leads to intriguing phenomena such as topological Anderson insulator, in which a metal or an ordinary insulator is transformed into a TI by disorder [35, 36]. Although in most studies, disorder is considered as a perturbation to the otherwise perfectly periodic structure, recent works have demonstrated canonical topological properties in quasiperiodic $37-39$ or even amorphous systems [40 43]. In light of these findings, complete classification of disordered TI has recently been worked out based on the noncommutative index theorem [44, 45]. These interesting results have significantly broadened the scope of TI and point to new directions in the search of novel topological materials.

In this paper, we further generalize the notion of topological materials by demonstrating a liquid state of Chern insulator within the framework of tight-binding molecular dynamics (TBMD) [46 48]. This novel liquid phase is characterized by a finite spectral gap and a nontrivial Bott index. We consider a 2D system of atoms with active $s$ and $p_{x}+i p_{y}$ orbitals. Inter-atomic potentials are parametrized based on the Slater-Koster scheme. At zero temperature, we show that the atoms crystalize into a triangular lattice whose band topology depends on the density. In particular, the lattice is characterized by a quantized non-zero Chern number at high densities. While the triangular lattice is melt by thermal fluctuations at finite temperature in $2 \mathrm{D}$, its nontrivial topology is transferred to the resultant liquid state which is characterized by a Bott index with the same value as the Chern number in the crystalline phase.

The tight-binding (TB) method has been used to construct several important lattice models for TI, such as the famous Haldane [6] and Kane-Mele model [7] on honeycomb lattice, and the Bernevig-Hughes-Zhang (BHZ) model for $\mathrm{HgTe}$ quantum well [10]. TB is also the main toolkit for constructing electronic models for disordered, quasi-periodic, and amorphous TIs. In all these systems, a TB Hamiltonian with appropriate parametrization is defined on a fixed network of sites representing the static atomic configuration. On the other hand, the atomic positions are dynamical variables in a liquid and their evolution is usually described using molecular dynamics (MD) simulation. In order to efficiently model the electronic structure of atomic liquids during the dynamical evolution of atoms, here we use the TBMD formulation [46]48] to construct a minimal model for liquid Chern insulator. TBMD is one of the so-called quantum 
MD techniques [51, 52, in which the atomic forces are obtained by solving the electron wavefunction quantum mechanically at every time-step. Importantly, the electronic structure effects are incorporated into the atomic dynamics in quantum MD methods.

We consider the following TBMD Hamiltonian,

$$
\begin{gathered}
\mathcal{H}=\sum_{i j} \sum_{\alpha \beta} t_{\alpha \beta}\left(\mathbf{r}_{i j}\right) c_{i \alpha}^{\dagger} c_{j \beta}+\sum_{i} \sum_{\alpha} \varepsilon_{\alpha} c_{i \alpha}^{\dagger} c_{i \alpha} \\
+\frac{1}{2} \sum_{i \neq j} \phi\left(\left|\mathbf{r}_{i j}\right|\right)+\sum_{i} \frac{\left|\mathbf{p}_{i}\right|^{2}}{2 m} .
\end{gathered}
$$

Here $c_{i \alpha}^{\dagger}$ is the creation operator of spin-polarized fermions of orbital $\alpha$ at the $i$-th atom, $t_{\alpha \beta}\left(\mathbf{r}_{i j}\right)$ is the hopping coefficient, $\phi(r)$ is a classical pair potential for the ions, $m$ is the mass of the ions, and $\mathbf{r}_{i j}=\mathbf{r}_{j}-\mathbf{r}_{i}$. The atomic positions $\mathbf{r}_{i}$ and momenta $\mathbf{p}_{i}$ are treated as classical degrees of freedom. The first two terms describe an effective TB model for a given atomic configuration $\left\{\mathbf{r}_{i}\right\}$ and represents the cohesive energy of the system. The third term corresponds to a repulsive energy that originates from the repulsion caused by overlapping orbitals. The last term is the kinetic energy of the atoms. The dynamics of the atoms is described by Newton equation of motion with forces computed quantum mechanically through the Hellman-Feynman theorem: $\mathbf{f}_{i}=-\sum_{j} \rho_{j \beta, i \alpha} \partial t_{\alpha \beta}\left(\mathbf{r}_{i j}\right) / \partial \mathbf{r}_{i}-\sum_{j} \partial \phi\left(\left|\mathbf{r}_{i j}\right|\right) / \partial \mathbf{r}_{i}$, where $\rho_{j \beta, i \alpha}=\left\langle c_{i \alpha}^{\dagger} c_{j \beta}\right\rangle$ is the electron density matrix solved from the TB model.

Next we describe a minimal 2D atomic system that exhibits nontrivial electronic topological properties. We assume spinless electrons occupying an $s$-like orbital (labeled by $\alpha=1)$ and a $p_{x}+i p_{y}$ orbital $(\alpha=2)$. The onsite orbital energies are denoted as $\varepsilon_{1,2}= \pm \Delta / 2$, where $\Delta$ is the energy splitting of the two orbitals. The hopping matrix can be parameterized using the Slater-Koster formula [49,

$$
t_{\alpha \beta}(\mathbf{r})=\left[\begin{array}{cc}
t_{s s}(r) & t_{s p}(r) e^{i \theta} \\
-t_{s p}(r) e^{-i \theta} & t_{p p}(r)
\end{array}\right],
$$

where $\mathbf{r}=(x, y)$ is the displacement vector between atoms, $r=|\mathbf{r}|$, and $\theta=\arctan (y / x)$ indicates the orientation. $t_{s s}, t_{s p}$, and $t_{p p}$ correspond to the $(s s \sigma),(s p \sigma)$, and $(p p \sigma)$ bond integrals [49, respectively. It is worth noting that same atomic orbitals are used in the $k \cdot p$ theory for the HgTe quantum well that exhibits quantum spin Hall effect [10.

In applications to real materials, the bond-length $r$ dependence of the hopping $t_{\alpha \beta}(r)$ and pair potential $\phi(r)$ is conventionally determined by fitting $a b$ initio calculation or experimental data to specific functional forms [50]. As a toy model to demonstrate the proof of principle, here we assume simple exponential decay for both functions: $t(r)=V \exp (-r / d)$ and $\phi(r)=\phi_{0} \exp (-r / \xi)$. More specifically, we assume the same characteristic length $d$ for the three $(s s \sigma),(s p \sigma)$, and $(p p \sigma)$ bonds. Specifically, we used $\xi=0.54 d$, and $\phi_{0}=33.4 \Delta, V_{s s}=-V_{p p}=V_{s p}=$

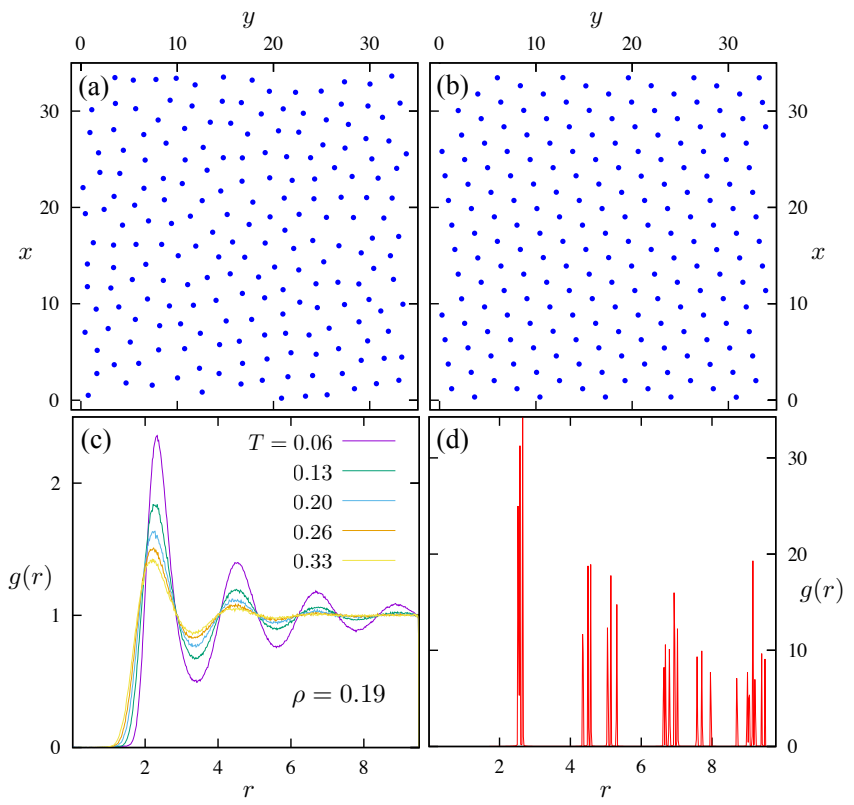

FIG. 1: (Color online) TBMD simulations of model Hamiltonian Eq. (1) with $N=200$ atoms and a fixed particle density $\rho=0.19$. Snapshots of atomic positions at temperatures $T=0.06$ and $T=10^{-5}$ are shown in (a) and (b), respectively. The thermal-averaged pair distribution functions $g(r)$ for the liquid and the (quasi) crystalline phases are shown in panels (c) and (d), respectively.

$4.2 \Delta$. In the following, we use $d$ and $\Delta$ for units of length and energy, respectively. We have checked that the existence of the liquid topological state does not depend sensitively on these parameters. However, the condition $V_{s s} V_{p p}<0$, which is satisfied in most materials, is crucial to the realization of topological phase. Finally, we assume half-filling for all simulations.

We performed our TBMD simulations using the standard velocity Verlet algorithm 53. The interatomic forces are computed by exactly diagonalizing the TB Hamiltonian at every time step. Fig. 1(a) and (b) show sample snapshots at temperatures $T=0.06$ and $T=10^{-5}$, respectively. The atoms at high temperatures are disordered. Yet they exhibit strong short-range correlation as evidenced from the radial distribution function $g(r)$ at finite temperatures shown in Fig. 1(c). Both the vanishing $g(r)$ for $r \lesssim 1.8$ and the oscillatory feature are characteristic of a liquid phase. As $T$ goes to zero, the atoms crystallize into a triangular lattice as shown in Fig. 1(b), which also manifests itself in the deltalike peaks of the corresponding $g(r)$ [Fig. 1(d)]. Strictly speaking, thermal fluctuations preclude any crystalline order in 2D. Indeed, the delta-like peaks disappear even at temperatures as low as $T \sim 10^{-3} \Delta$. However, it is appropriate to consider local crystalline order at low enough temperatures when the thermal correlation length $\xi_{T}$ is much larger than the system size.

The crystallization of atoms at $T \rightarrow 0$ makes it easier to analyze their topological properties using conventional 
(a)

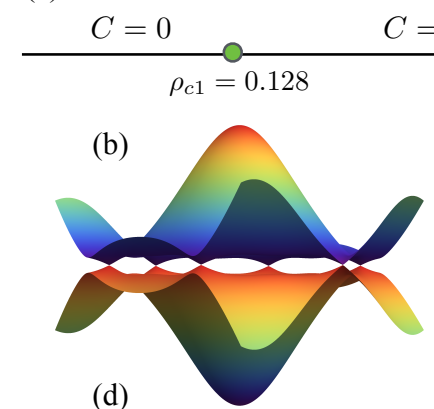

(d)

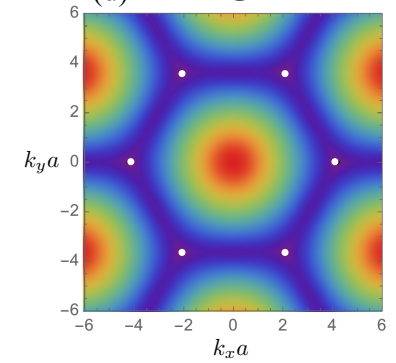

(e)

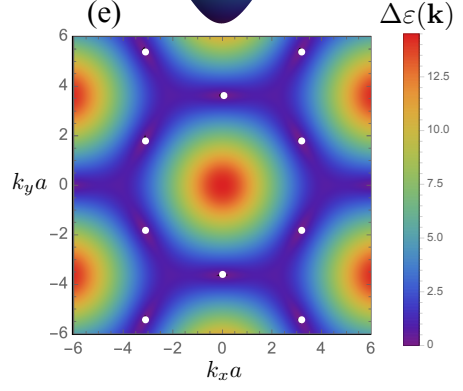

FIG. 2: (Color online) (a) phase diagram showing two quantum critical points separating three topologically distinct phases in the triangular-lattice TB model. Panels (b) and (c) show the band structures at the two critical densities $\rho_{c 1}$ and $\rho_{c 2}$, respectively. Six Dirac nodes develop at (b) the corners (two inequivalent $K$ points) of the BZ, and (c) the midpoints of BZ edges (three inequivalent $M$ points). The $\mathbf{k}$-dependent band-gap $\Delta \varepsilon(\mathbf{k})=2|\mathbf{m}(\mathbf{k})|$ at the two critical densities are shown in (d) and (e), respectively. The white dots mark the Dirac points where $\Delta \varepsilon=0$.

band theory toolkits. First, the lattice TB model can be easily solved for rather large lattices using the Fourier transform method. The TB Hamiltonian in momentum space reads $H_{\alpha \beta}(\mathbf{k})=\sum_{\mathbf{R}} t_{\alpha \beta}(\mathbf{r}) \exp (i \mathbf{k} \cdot \mathbf{R})$, where $\mathbf{R}$ runs over the triangular lattice sites up to a large cutoff $r_{c}=20$. To obtain its spectrum and topological index, we express the $2 \times 2$ Hamiltonian in terms of the Pauli matrices: $H(\mathbf{k})=\varepsilon_{0}(\mathbf{k}) \mathbb{I}+\mathbf{m}(\mathbf{k}) \cdot \boldsymbol{\sigma}$, where $\mathbb{I}$ is a $2 \times 2$ identity matrix. Its spectrum consists of two bands with dispersions readily computed as: $\varepsilon_{ \pm}(\mathbf{k})=\varepsilon_{0}(\mathbf{k}) \pm|\mathbf{m}(\mathbf{k})|$. In most cases, the two bands do not overlap and are separated by a direct energy gap $\varepsilon_{g}=2 \min _{\mathbf{k}}|\mathbf{m}(\mathbf{k})|$. At half-filling, the lower energy band $\varepsilon_{-}(\mathbf{k})$ is completely filled. Our explicit numerical calculation shows that the band-gap closes at two critical densities, at which lowenergy Dirac cones emerge at the high-symmetry points of the Brillouin zone (BZ) as shown in Fig. 2.

The two critical points $\rho_{c 1,2}$ also separate phases of different topologies. In the gapped phase, the Chern number of the occupied band is given by the integral over the BZ: $C=(1 / 4 \pi) \iint \hat{\mathbf{m}} \cdot \partial_{k_{x}} \hat{\mathbf{m}} \times \partial_{k_{y}} \hat{\mathbf{m}} d k_{x} d k_{y}$. At low densities $\rho<\rho_{c 1}$, we find a trivial band insulator with $C=0$. The band-gap closes at the first critical density $\rho_{c 1}$ as Dirac cones develop at the six corners of the BZ. Only two of these six Dirac nodes $\left(K\right.$ and $\left.K^{\prime}\right)$ are inequivalent. Further increasing the density gaps out these two Dirac nodes, giving rise to a topological insulator with $C=-2$. As we further increase the density, the atoms undergo another quantum phase transition at $\rho_{c 2}$ characterized again by appearance of Dirac cones, this time at the midpoints of BZ edges; these correspond to three inequivalent $M$ points. Gapping out these three Dirac nodes increases the Chern number by 3 , leading to another topological insulating phase with $C=+1$.

Having described the topological properties of the crystalline phase in the $T \rightarrow 0$ limit, one natural question that arises is how the nontrivial band topology is transferred to the liquid state at finite temperatures. To answer this question, we first compute the average energy gap of the atomic fluid. For a system with $N$ atoms, solving the TB Hamiltonian in Eq. (1) gives $2 N$ eigenenergies $\varepsilon_{m}(m=1,2, \cdots, 2 N)$. Assuming these energies are in ascending order, we define an energy gap at halffilling as $\varepsilon_{g}=\varepsilon_{N+1}-\varepsilon_{N}$. We note that at finite $T$, there are always thermal excitations of electrons above the gap, so this $\varepsilon_{g}$ should be viewed as the intrinsic energy gap of the electron spectrum, in the same spirit as the definition of band-gap for a crystalline state. A crucial difference is that a configurational average is required in order to obtain an effective energy gap for the liquid phase.

Fig. 3(a) shows the density dependence of the energy gap $\varepsilon_{g}$ averaged over a large number of atomic configurations in the liquid state at different temperatures. Also shown for comparison is the curve for the crystalline phase. As discussed above, $\varepsilon_{g}$ vanishes at two critical densities $\rho_{c 1,2}$ in the presence of crystalline order. In the liquid phase, the energy gap becomes very small for a finite range of density around $\rho_{c 1}$, indicating an extended regime of liquid metal phase. Moreover, the size of this metallic regime increases with increasing temperature. On the other hand, a nonzero gap persists at small and large densities even in the liquid state.

To describe the nontrivial topology of the atomic liquid, we compute a topological invariant called the Bott index [54, which plays a similar role as the Chern number for crystalline state. Indeed, the Bott index reduces to Chern number in the presence of long-range crystalline order. To compute this index for a given atomic configuration $\left\{\mathbf{r}_{i}=\left(x_{i}, y_{i}\right)\right\}$, we first construct two diagonal matrices $\Theta$ and $\Phi$, such that $\Theta_{i i}=2 \pi x_{i} / L_{x}$ and $\Phi_{i i}=2 \pi y_{i} / L_{y}$. Next we compute the band-projected position matrices $U$ and $V$ according to $U=P \exp (i \Theta) P$ and $V=P \exp (i \Phi) P$, where $P$ is the projector to the lowest $N$ eigen-states, corresponding to the (half) occupied states at zero temperature. The Bott index is then given by $\nu=(1 / 2 \pi) \operatorname{Im}\left\{\operatorname{tr}\left[\log \left(V U V^{\dagger} U^{\dagger}\right)\right]\right\}$ which only takes on integer values [54]. Essentially, $\nu$ quantifies the topological obstructions to the existence of localized Wannier functions spanning the occupied manifold [54].

By averaging over a large number of atomic configurations from our TBMD simulations, we compute the Bott index as a function of density for various temperatures in the liquid phase. The results are summarized in Figs. 3(b) and (d). Also shown for comparison is the nu- 

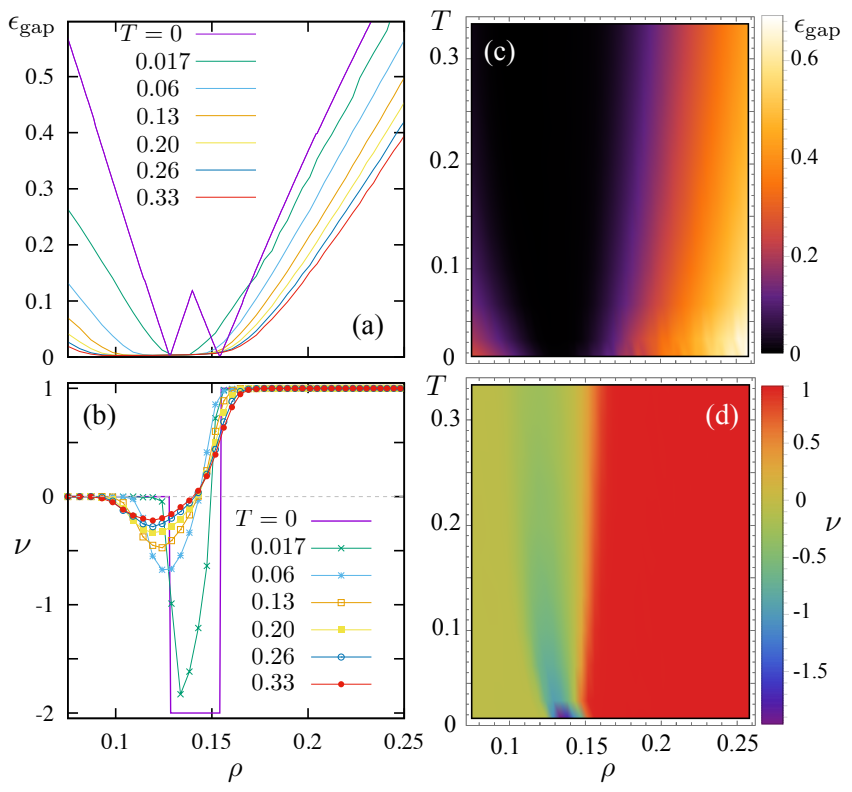

FIG. 3: (Color online) (a) Energy gap $\varepsilon_{g}$ as a function of density for varying temperatures in the liquid phase. Also shown for comparison is the band-gap of the crystalline phase obtained using Fourier transform method. (b) Configurationaveraged Bott index $\nu$ vs density $\rho$ for varying temperatures. The $T=0$ curve is the Chern number $C$ of the triangularlattice TB model. Panels (c) and (d) show the density plot of averaged energy gap and Bott index in the $T$ - $\rho$ plane.

merical Chern number of the crystalline state. At small and large densities, the averaged $\nu$ exhibits quantized values at 0 and 1 , respectively. Taking into account that a finite gap exists also in these two regimes, we conclude that the atomic liquid is a trivial band insulator at small density, and a topological Chern insulator at large $\rho$. In fact, we find that $\nu$ is exactly quantized at 0 and 1 for small and large $\rho$, respectively, for all snapshots in our MD simulations. For intermediate values of $\rho$, the Bott index fluctuates between different integer values, as illustrated in Fig. 4, giving rise to a non-quantized $\nu$ after average. This intermediate phase partially inherits the nontrivial topology of the crystalline state with a Chern number $C=-2$ and can be viewed as a topological liquid quasi-metal. Its electronic structure might be similar to the diffusive metal phase induced by strong disorder in topological semimetals [28 34].

Our two-orbital TBMD model provides the first example of a Chern insulator or quantum anomalous Hall insulator in an atomic liquid. One can use this model to construct a liquid state of quantum spin-Hall (QSH) insulator similar to how Kane-Mele model is built from the Haldane model [7. Since time-reversal symmetry is required for the QSH effect, we add spins to the electrons and consider two copies of our atomic model: one with $|s, \uparrow\rangle$ and $\left|p_{x}+i p_{y}, \uparrow\right\rangle$ spin-orbital states, while the other with $|s, \downarrow\rangle$ and $\left|p_{x}+i p_{y}, \downarrow\right\rangle$. Again, the same atomic orbitals are used in the BHZ model for the quantum spin-

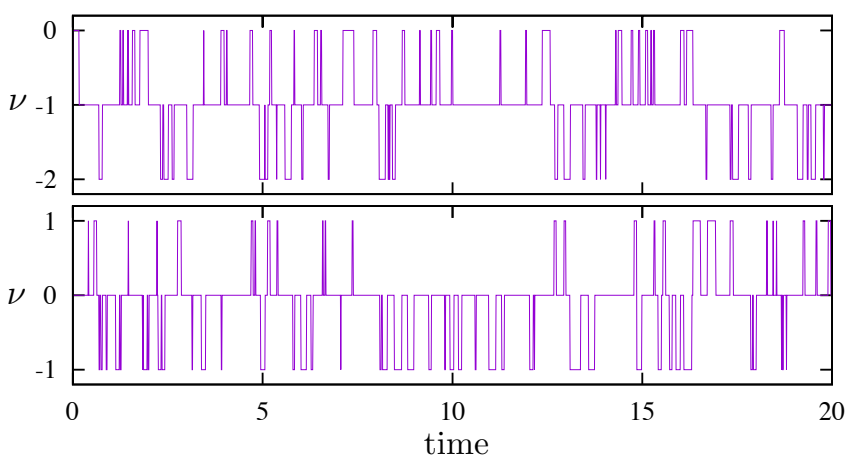

FIG. 4: (Color online) The instantaneous Bott index as a function of time for (a) $\rho=0.133$ and (b) $\rho=0.143$ from TBMD simulations with temperature $T=0.017$.

Hall insulator in HgTe quantum wells [10. This simple model with two decoupled copies related by time-reversal symmetry gives a quantized spin-Hall conductivity. In the presence of small spin-nonconserving perturbations, the topological nature of the quantum spin-Hall state ensures the existence of spin-filtered edge states as long as time-reversal symmetry is preserved. Construction of 3D liquid TI will be left for future studies.

To summarize, we have demonstrated a novel liquid topological insulator based on the TBMD formulation. This new state of matter generalizes the notion of topological materials, and offers intriguing technological possibilities. Together with the recent reports of TI in amorphous systems 41 43, our work further shows the robust nature of topological insulators, which does not rely on the existence of a well defined BZ. Indeed, the amorphous TI phase can be viewed as the high- $T$ "gas" phase, characterized by a flat radial distribution function, of our atomic system. Given the fact that TI can exist even in a set of randomly connected points [41, the possibility of a liquid TI might not be a complete surprise. However, it is unclear whether the topological properties survive once these "points" or atoms are allowed to move around. For example, the rearrangement of atoms might close the electronic gap, rendering the system a liquid metal. Our proof of principle study shows that nontrivial topology of electronic structure can exist in a dynamical liquid state.

Our work also suggests that systematic searches for liquid or amorphous TIs can be attained by the TBMD approach. Over the past few decades, significant progress has been made toward TBMD modeling of realistic materials such as transition metals, semiconducting alloys, and liquid chalcogenides [55, 56, especially with more accurate parameterization of the radial hopping functions and pair potentials [50]. However, the effects of spinorbit coupling, which is crucial to topological properties, have yet to be systematically investigated in the TBMD framework. An important step towards this goal is the TBMD parametrization of systems containing $4 d$ or $5 d$ atoms, which play an key role in most topological materials. Interestingly, several well-studied TI compouds, 
such as $\mathrm{Bi}_{2} \mathrm{Te}_{3}, \mathrm{Sb}_{2} \mathrm{Te}_{3}, \mathrm{Ag}_{2} \mathrm{Te}, \mathrm{Ag}_{2} \mathrm{Se}$, and $\mathrm{PbTe}$, are listed as liquid semiconductors in their molten state [57. Such liquid alloys exhibit semiconducting behaviors and are characterized by short-range order and a well defined chemical stoichiometry. It is intriguing to see whether these compounds are potential liquid TI through careful $a b$ initio MD simulations [52] and characterization. We hope that our work will motivate further numerical and experimental search of such liquid TI in real materials.
Acknowledgements. I thank Jeffrey Teo, D. Louca, and U. Chatterjee for insightful discussions on disordered topological insulators and collaborations on related projects. This work is partially supported by the Center for Materials Theory as a part of the Computational Materials Science (CMS) program, funded by the US Department of Energy, Office of Science, Basic Energy Sciences, Materials Sciences and Engineering Division.
[1] M. Z. Hasan and C. L. Kane, Topological insulators, Rev. Mod. Phys. 82, 3045 (2010).

[2] X.-L. Qi and S.-C. Zhang, Topological insulators and superconductors, Rev. Mod. Phys. 83, 1057 (2011).

[3] K. v. Klitzing, G. Dorda, and M. Pepper, New method for high-accuracy determination of the fine-structure constant based on quantized hall resistance, Phys. Rev. Lett. 45, 494 (1980).

[4] D. J. Thouless, M. Kohmoto, M. P. Nightingale, and M. den Nijs, Quantized Hall Conductance in a TwoDimensional Periodic Potential, Phys. Rev. Lett. 49, 405 (1982).

[5] J. E. Avron, R. Seiler, and B. Simon, Homotopy and Quantization in Condensed Matter Physics, Phys. Rev. Lett. 51, 51 (1983).

[6] F. D. M. Haldane, Model for a Quantum Hall Effect without Landau Levels: Condensed-Matter Realization of the "Parity Anomaly", Phys. Rev. Lett. 61, 2015 (1988).

[7] C. L. Kane and E. J. Mele, $Z_{2}$ Topological Order and the Quantum Spin Hall Effect, Phys. Rev. Lett. 95, 146802 (2005).

[8] C. L. Kane and E. J. Mele, Quantum Spin Hall Effect in Graphene, Phys. Rev. Lett. 95, 226801 (2005).

[9] B. A. Bernevig and S.-C. Zhang, Quantum Spin Hall Effect, Phys. Rev. Lett. 96, 106802 (2006).

[10] B. A. Bernevig, T. L. Hughes, and S.-C. Zhang, Quantum Spin Hall Effect and Topological Phase Transition in HgTe Quantum Wells, Science 314, 1757 (2006).

[11] M. König, S. Wiedmann, C. Brne, A. Roth, H. Buhmann, L. W. Molenkamp, X.-L. Qi, and S.-C. Zhang, Quantum Spin Hall Insulator State in HgTe Quantum Wells, Science 318, 766 (2007).

[12] L. Fu, C. L. Kane, and E. J. Mele, Topological Insulators in Three Dimensions, Phys. Rev. Lett. 98, 106803 (2007).

[13] J. E. Moore and L. Balents, Topological invariants of time-reversal-invariant band structures, Phys. Rev. B 75, 121306 (2007).

[14] R. Roy, Topological phases and the quantum spin Hall effect in three dimensions, Phys. Rev. B 79, 195322 (2009).

[15] D. Hsieh, D. Qian, L. Wray, Y. Xia, Y. S. Hor, R. J. Cava, and M. Z. Hasan, A topological Dirac insulator in a quantum spin Hall phase, Nature (London) 452, 970 (2008).

[16] X.-L. Qi, T. L. Hughes, and S.-C. Zhang, Topological field theory of time-reversal invariant insulators, Phys. Rev. B 78, 195424 (2008).

[17] A. P. Schnyder, S. Ryu, A. Furusaki, and A. W. W. Ludwig, Classification of topological insulators and superconductors in three spatial dimensions, Phys. Rev. B 78, 195125 (2008)
[18] A. Kitaev, Periodic table for topological insulators and superconductors, AIP Conf. Proc. 1134, 22 (2008).

[19] S. Ryu, A. P. Schnyder, A. Furusaki, A. W. W. Ludwig, Topological insulators and superconductors: tenfold way and dimensional hierarchy, New J. Phys. 12, 065010 (2010).

[20] A. Altland and M. R. Zirnbauer, Nonstandard symmetry classes in mesoscopic normal-superconducting hybrid structures, Phys. Rev. B 55, 1142 (1997).

[21] D. N. Sheng and Z. Y. Weng, Disappearance of Integer Quantum Hall Effect, Phys. Rev. Lett. 78, 318 (1997).

[22] M. Onoda, Y. Avishai, and N. Nagaosa, Localization in a Quantum Spin Hall System, Phys. Rev. Lett. 98, 076802 (2007).

[23] R. S. K. Mong, J. H. Bardarson, and J. E. Moore, Quantum Transport and Two-Parameter Scaling at the Surface of a Weak Topological Insulator, Phys. Rev. Lett. 108, 076804 (2012).

[24] Z. Ringel, Y. E. Kraus, and A. Stern, Strong side of weak topological insulators, Phys. Rev. B 86, 045102 (2012).

[25] G. Schubert, H. Fehske, L. Fritz, and M. Vojta, Fate of topological-insulator surface states under strong disorder, Phys. Rev. B 85, 201105(R) (2012).

[26] K. Kobayashi, T. Ohtsuki, and K.-I. Imura, Disordered Weak and Strong Topological Insulators, Phys. Rev. Lett. 110, 236803 (2013)

[27] S. Liu, T. Ohtsuki, and R. Shindou, Effect of Disorder in a Three-Dimensional Layered Chern Insulator, Phys. Rev. Lett. 116, 066401 (2016).

[28] P. Goswami and S. Chakravarty, Quantum Criticality between Topological and Band Insulators in 3+1 Dimensions, Phys. Rev. Lett. 107, 196803 (2011).

[29] S. Ryu and K. Nomura, Disorder-induced quantum phase transitions in three-dimensional topological insulators and superconductors, Phys. Rev. B 85, 155138 (2012).

[30] K. Kobayashi, T. Ohtsuki, K.-I. Imura, and I. F. Herbut, Density of States Scaling at the Semimetal to Metal Transition in Three Dimensional Topological Insulators, Phys. Rev. Lett. 112, 016402 (2014).

[31] J. H. Pixley, P. Goswami, and S. Das Sarma, Anderson Localization and the Quantum Phase Diagram of Three Dimensional Disordered Dirac Semimetals, Phys. Rev. Lett. 115, 076601 (2015).

[32] C.-Z. Chen, J. Song, H. Jiang, Q.-F. Sun, Z. Wang, and X. C. Xie, Disorder and Metal-Insulator Transitions in Weyl Semimetals, Phys. Rev. Lett. 115, 246603 (2015).

[33] H. Shapourian and T. Hughes, Phase diagrams of disordered Weyl semimetals, Phys. Rev. B 93, 075108 (2016).

[34] S. V. Syzranov and L. Radzihovsky, High-Dimensional Disorder-Driven Phenomena in Weyl Semimetals, Semi- 
conductors, and Related Systems, Annu. Rev. Condens. Matter Phys. 9, 35 (2017).

[35] J. Li, R.-L. Chu, J. K. Jain, and S.-Q. Shen, Topological Anderson Insulator, Phys. Rev. Lett. 102, 136806 (2009).

[36] H.-M. Guo, G. Rosenberg, G. Refael, and M. Franz, Topological Anderson Insulator in Three Dimensions, Phys. Rev. Lett. 105, 216601 (2010).

[37] Y. E. Kraus, Y. Lahini, Z. Ringel, M. Verbin, and O. Zilberberg, Topological States and Adiabatic Pumping in Quasicrystals, Phys. Rev. Lett. 109, 106402 (2012).

[38] I. C. Fulga, D. I. Pikulin, and T. A. Loring, Aperiodic Weak Topological Superconductors, Phys. Rev. Lett. 116, 257002 (2016).

[39] M. A. Bandres, M. C. Rechtsman, and M. Segev, Topological Photonic Quasicrystals: Fractal Topological Spectrum and Protected Transport, Phys. Rev. X 6, 011016 (2016).

[40] G.-W. Chern, A. Rahmani, I. Martin, C. D. Batista, Quantum Hall ice, Phys. Rev. B 90, 241102 (2014).

[41] A. Agarwala and V. B. Shenoy, Topological Insulators in Amorphous Systems, Phys. Rev. Lett. 118, 236402 (2017).

[42] N. P. Mitchell, L. M. Nash, D. Hexner, A. M. Turner, and W. T. M. Irvine, Amorphous topological insulators constructed from random point sets, Nat. Phys. 14, 380 (2018).

[43] K. Pöyhönen, I. Sahlberg, A. Westström, and T. Ojanen, Amorphous topological superconductivity in a Shiba glass, Nat. Commun. 9, 2103 (2018).

[44] E. Prodan, H. Schulz-Baldes, Bulk and boundary invariants for complex topological insulators: From K-theory to physics, Springer, Berlin, 2016.

[45] H. Katsura and T. Koma, The noncommutative index theorem and the periodic table for disordered topological insulators and superconductors, J. Math. Phys. 59,
031903 (2018).

[46] F. S. Khan and J. Q. Broughton, Simulation of silicon clusters and surfaces via tight-binding molecular dynamics, Phys. Rev. B 39, 3688 (1989).

[47] C. Z. Wang, C. T. Chan, and K. M. Ho, Empirical tightbinding force model for molecular-dynamics simulation of Si, Phys. Rev. B 39, 8586 (1989).

[48] S. Goedecker and L. Colombo, Efficient Linear Scaling Algorithm for Tight-Binding Molecular Dynamics, Phys. Rev. Lett. 73, 122 (1994).

[49] J. C. Slater and G. F. Koster, Simplified LCAO Method for the Periodic Potential Problem, Phys. Rev. 94, 1498 (1954).

[50] L. Goodwin, A. J. Skinner, and D. G. Pettifor, Generating Transferable Tight-Binding Parameters: Application to Silicon, Europhys. Lett. 9, 701 (1989).

[51] R. Car and M. Parrinello, Unified Approach for Molecular Dynamics and Density-Functional Theory, Phys. Rev. Lett. 55, 2471 (1985).

[52] D. Marx and J. Hutter, Ab Initio Molecular Dynamics, Cambridge University Press (Cambridge, 2009).

[53] M. P. Allen and D. J. Tildesley, Computer Simulation of Liquids (Oxford Science, Oxford, 1987).

[54] T. A. Loring and M. B. Hastings, Disordered topological insulators via $C^{*}$-algebras, Europhys. Lett. 92, 67004 (2010).

[55] F. Cleri and V. Rosato, Tight-binding potentials for transition metals and alloys, Phys. Rev. B 48, 22 (1993).

[56] C. M. Goringe, D. R. Bowler, and E. Hernández, Tightbinding modeling of materials, Rep. Prog. Phys. 60, 1447 (1997).

[57] J. E. Enderby and A. C. Barnes, Liquid semiconductors, Rep. Prog. Phys. 53, 85 (1990). 